Acta Crystallographica Section E

Structure Reports

Online

ISSN 1600-5368

Andrew Parkin,* Suzanne M.

Harte, Donnie Carmichael, Scott

Currie, Lorna Drummond, Adam

Haahr, Kevin Haggarty, Tracey

Hunter, André Lamarque,

Loretta Lawton, Craig Martin,

Jennifer E. Mathieson,

Jennifer S. Mathieson, Thomas

McGlone, Julie McGregor, Liam

McMillan, Louise Robertson,

Robert Thatcher, Steven Vance

and Chick C. Wilson

Department of Chemistry, University of Glasgow, University Avenue, Glasgow

G12 8QQ, Scotland

Correspondence e-mail:

a.parkin@chem.gla.ac.uk

\section{Key indicators}

Single-crystal X-ray study

$T=293 \mathrm{~K}$

Mean $\sigma(\mathrm{C}-\mathrm{C})=0.003 \AA$

$R$ factor $=0.054$

$w R$ factor $=0.164$

Data-to-parameter ratio $=17.3$

For details of how these key indicators were automatically derived from the article, see http://journals.iucr.org/e.

\title{
2-Phenoxybenzoic acid at room temperature
}

In the crystal structure of the title compound, $\mathrm{C}_{13} \mathrm{H}_{10} \mathrm{O}_{3}$, the molecules form classical hydrogen-bonded carboxylic acid dimers $[\mathrm{O} \cdots \mathrm{O}=2.651$ (2) $\AA$ ] . These dimers are linked by $\mathrm{C}-$ $\mathrm{H} \cdots \pi$ and $\pi-\pi$ interactions to give a three-dimensional network.

\section{Comment}

Benzoic acid is a compound that has an elegant simplicity to its molecular structure, but its derivatives display an enormous complexity and diversity of molecular structures. The latest version of the Cambridge Structural Database (CSD, Version 5.26; Allen, 2002) contains 1883 structures with a benzoic acid derivative existing in the crystal structure as an isolated molecule; this does not include structures in which the molecules are either deprotonated or coordinated to metal ions. By contrast, the simple and readily available title compound, (I), is only observed in four crystal structures in the CSD, and in all of these it serves as a ligand. The 3- and 4-phenoxybenzoic acid structures are observed even less frequently, with zero and one structures of these compounds, respectively. Possibly the most closely related structure available in the CSD is that of 2-(2-carboxyphenoxy)benzoic acid (CSD refcode MIGPAT; Field \& Venkataraman, 2002), which differs only by the presence of an extra carboxylic acid group on the second benzene ring.

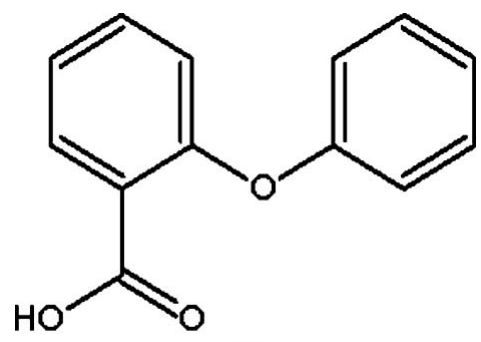

(I)

The molecular geometry observed in the structure of (I) is mostly unremarkable, with the principal features of note being the prolate displacement ellipsoid of atom O10, which is consistent with a large vibration perpendicular to the plane of the benzoic acid fragment (Fig. 1). This motion is not obviously propagated in the second benzene ring; in this portion, the displacement ellipsoids are surprisingly close to spherical, although large. These observations are most likely due to the combination of three movements: a typical in-plane rotational movement around the ring, the translational movement observed for $\mathrm{O} 10$ in the plane of this ring and perpendicular to the $\mathrm{O} 10-\mathrm{C} 11$ bond vector, and a rotational
Received 24 May 2005 Accepted 21 June 2005 Online 30 June 2005 


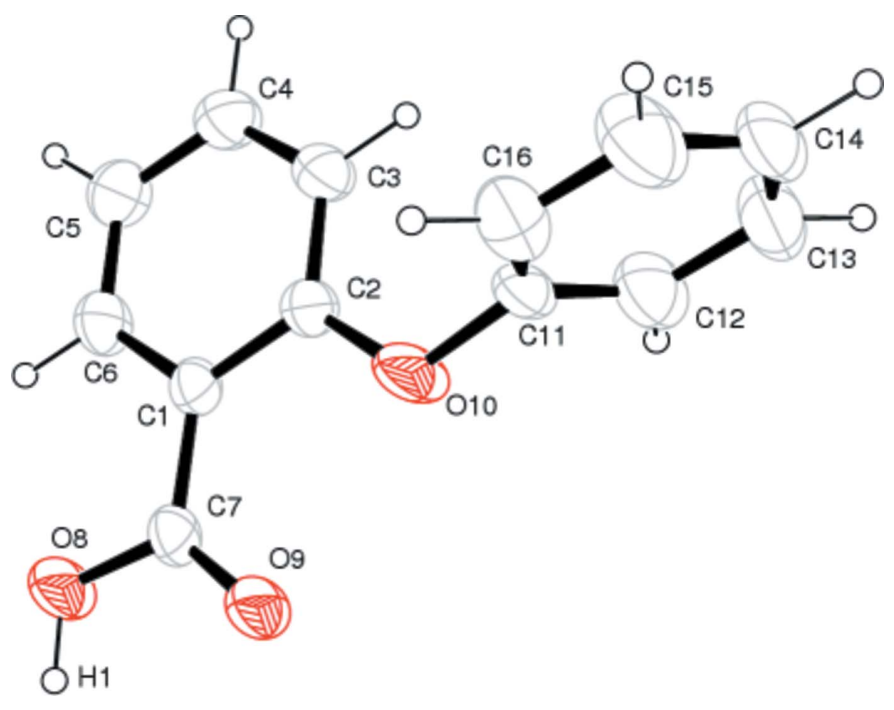

Figure 1

A drawing of the molecule of (I), showing the atomic numbering scheme. Ellipsoids for non- $\mathrm{H}$ atoms are shown at the $30 \%$ probability level. All $\mathrm{H}$ atoms take their number from the parent $\mathrm{C}$ atom, except for $\mathrm{H} 1$.

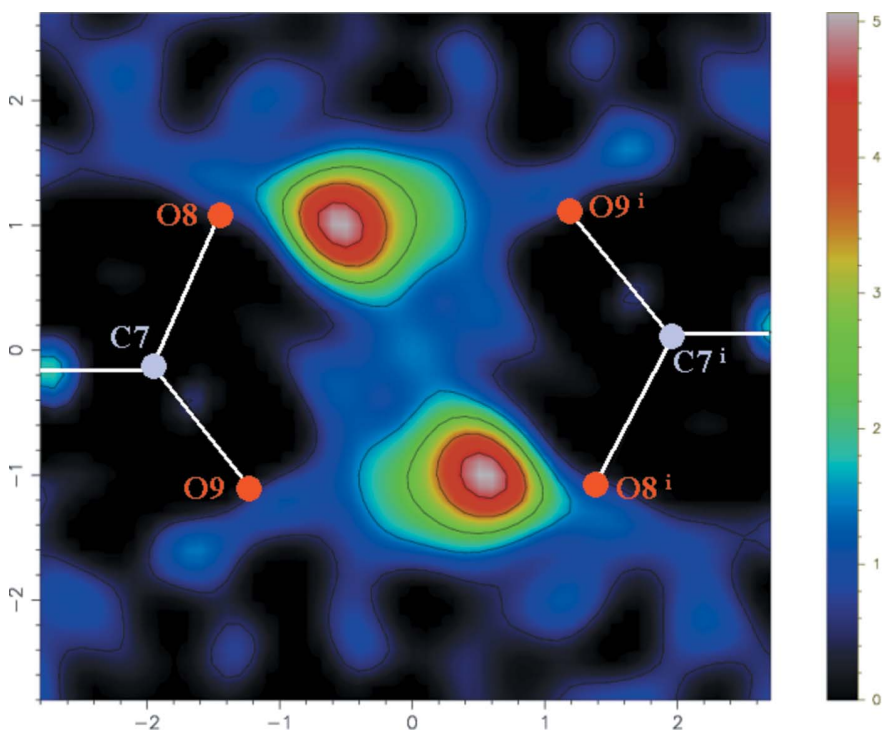

Figure 2

Fourier difference map section through the carboxylic acid dimer plane defined by atoms $\mathrm{C} 7, \mathrm{O} 8, \mathrm{O} 9, \mathrm{C}^{\mathrm{i}}, \mathrm{O}^{\mathrm{i}}$ and $\mathrm{O} 9^{\mathrm{i}}$ [symmetry code: (i) $3-x$, $1-y,-z]$. There is clearly only a single peak associated with each hydrogen bond, corresponding to an ordered $\mathrm{H}$ atom.

movement around the $\mathrm{O} 10-\mathrm{C} 11$ bond vector. The average $\mathrm{C}-\mathrm{C}$ bond length in this ring is slightly short, at $1.36 \AA$; this bond shortening can also be attributed to the effect of large thermal libration. The normals to the planes of the two benzene rings are nearly perpendicular, at $89.8(2)^{\circ}$.

The molecules of (I) assemble to form a classical hydrogenbonded dimer, in which the $\mathrm{C} 7-\mathrm{O} 9$ and $\mathrm{C} 7-\mathrm{O} 8$ bond lengths in the carboxylic acid group of 1.223 (2) and 1.3015 (18) $\AA$, respectively, indicate a well ordered hydrogen bond. This is supported by the lack of $\mathrm{H}$-atom disorder observed in the Fourier difference map (calculated with the program

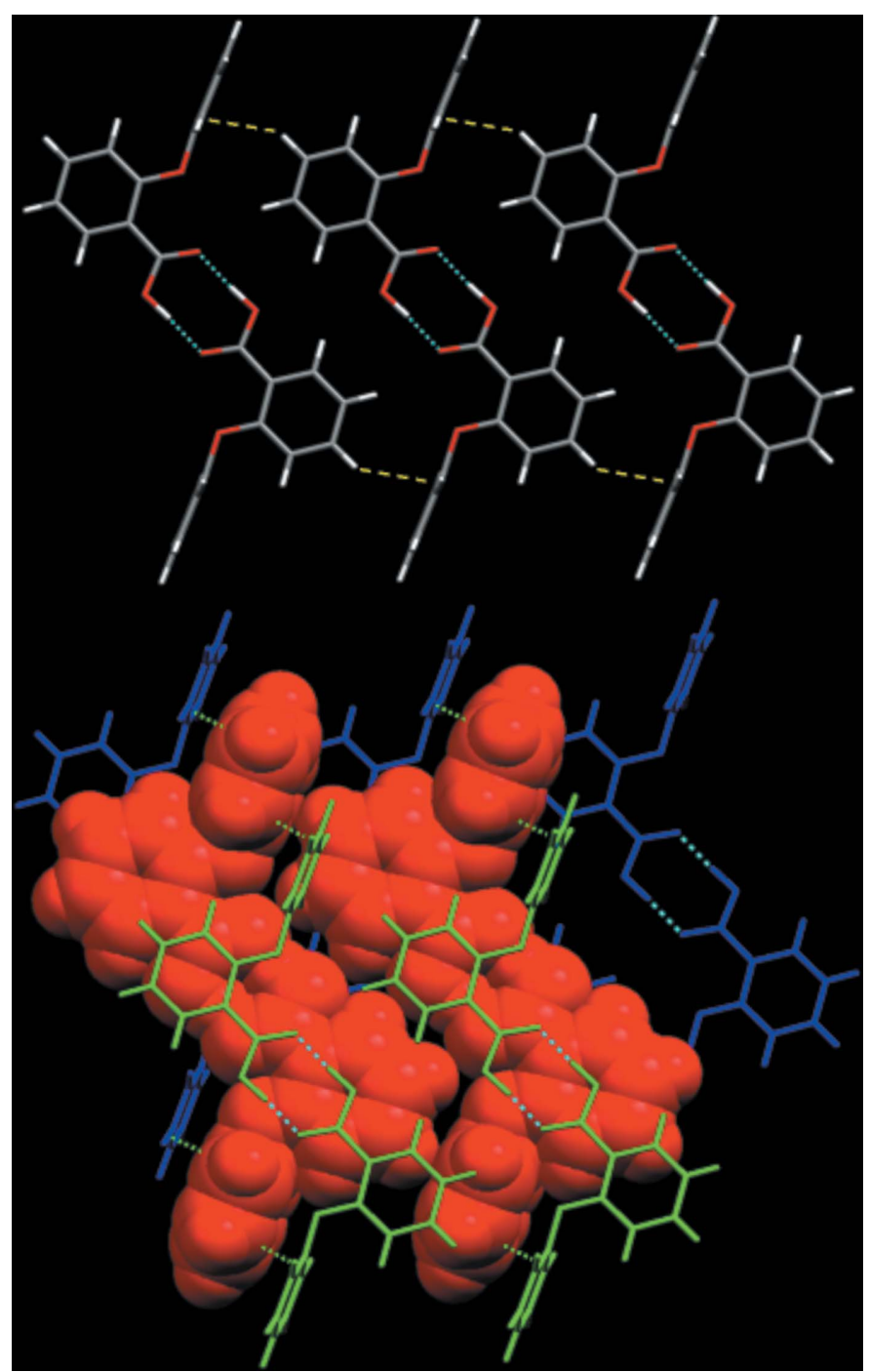

Figure 3

Packing plots of (I), illustrating the principal contacts in the structure. (top) The hydrogen-bond dimers link together into ribbons via $\mathrm{C}-\mathrm{H} \cdots \pi$ contacts (shown in yellow). (bottom) These ribbons stack due to $\pi-\pi$ interactions (shown in pale green).

MAPVIEW, part of the WinGX suite; Farrugia, 1999) through the dimer group (Fig. 2). The single crystallographically

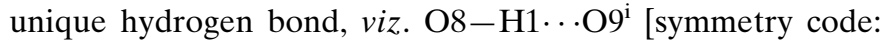
(i) $3-x, 1-y,-z]$, exhibits a typical $\mathrm{O} \cdots \mathrm{O}$ separation for benzoic acid dimers of 2.651 (2) $\AA$. The remainder of the contacts lie outside the sum of the van der Waals radii of the two atoms involved, but these very weak interactions can still be used to describe the remainder of the structure. The dimers assemble into extended ribbons through $\mathrm{C}-\mathrm{H} \cdots \pi$ interactions of $3.658 \AA$ for $\mathrm{C} 4-\mathrm{H} 4 \cdots \mathrm{C} 12^{\mathrm{ii}}$ [symmetry code: (ii) $x$, $y-1, z]$ (Fig. 3a), and these ribbons form stacks defined by a $\pi-\pi$ contact of $3.446 \AA$ between atoms $\mathrm{C} 13$ and $\mathrm{C} 16^{\mathrm{iii}}$ [symmetry code: (iii) $x-1, y, z$ ] (Fig. $3 b$ ). The stacks pack together with $\mathrm{C}-\mathrm{H} \cdots \pi$ interactions of $3.697 \AA$ for $\mathrm{C} 12-$ H12 $\cdots C 5^{\text {iv }}$ [symmetry code: (iv) $x-1, y+1, z$ ] (Fig. 4).

The most striking difference between the molecular structure presented here and that of MIGPAT (Field \& Venkata- 


\section{organic papers}

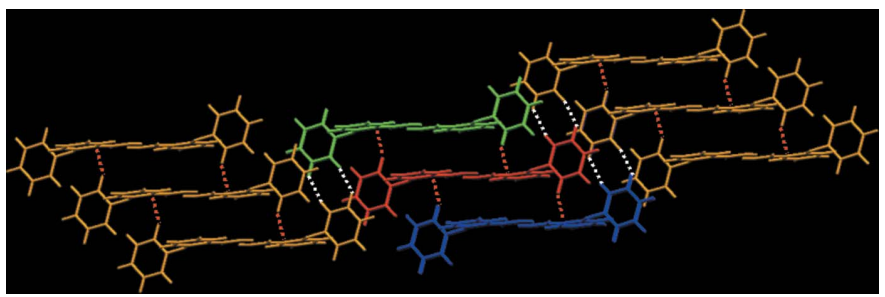

Figure 4

A packing plot of the entire structure. The stacks are linked by $\mathrm{C}-\mathrm{H} \cdots \pi$ interactions, shown in red.

raman, 2002) is the geometry of the carboxylic acid group. In the title compound, it is clear from the bond lengths that the $\mathrm{C}=\mathrm{O}$ double bond is $\mathrm{C} 7=\mathrm{O} 9$, involving the $\mathrm{O}$ atom closest to the ether group. By contrast, the shorter $\mathrm{C}-\mathrm{O}$ bond in MIGPAT is that further from the ether $\mathrm{O}$ atom, although the difference between the two bond lengths is much less than we report here. As the two chemically different carboxylic acids in MIGPAT are crystallographically identical, it is possible that there is some correlated structural disorder between the $\mathrm{C}-\mathrm{O}$ and $\mathrm{C}=\mathrm{O}$ bonds; this might explain the very similar $\mathrm{C}-\mathrm{O}$ bond lengths in MIGPAT.

\section{Experimental}

The title compound was used as received from Aldrich. Crystals of diffraction quality were grown from an acetone solution.

\section{Crystal data \\ $\mathrm{C}_{13} \mathrm{H}_{10} \mathrm{O}_{3}$ \\ $M_{r}=214.22$ \\ Triclinic, $P \overline{1}$ \\ $a=5.2736(5) \AA$ \\ $b=7.7366(6) \mathrm{A}$ \\ $c=13.6863(10) \AA$ \\ $\alpha=89.184(6)$ \\ $\beta=83.433(6)^{\circ}$ \\ $\gamma=74.640(6)$ \\ $V=534.84(8) \AA^{3}$}

\author{
$Z=2$ \\ $D_{x}=1.330 \mathrm{Mg} \mathrm{m}^{-3}$ \\ Mo $K \alpha$ radiation \\ Cell parameters from 8180 \\ reflections \\ $\theta=2-28^{\circ}$ \\ $\mu=0.10 \mathrm{~mm}^{-1}$ \\ $T=293 \mathrm{~K}$ \\ Block, colourless \\ $0.30 \times 0.15 \times 0.10 \mathrm{~mm}$
}

\section{Data collection}

Bruker APEX2 diffractometer $\varphi$ and $\omega$ scans

Absorption correction: multi-scan

(SADABS; Sheldrick, 1996)

$T_{\min }=0.98, T_{\max }=0.99$

8180 measured reflections

2565 independent reflections

\section{Refinement}

Refinement on $F^{2}$

$R\left[F^{2}>2 \sigma\left(F^{2}\right)\right]=0.054$

$w R\left(F^{2}\right)=0.164$

$S=0.93$

2565 reflections

148 parameters

$\mathrm{H}$ atoms treated by a mixture of independent and constrained refinement
1516 reflections with $I>2 \sigma(I)$

$R_{\text {int }}=0.028$

$\theta_{\text {max }}=28.5^{\circ}$

$h=-6 \rightarrow 7$

$k=-10 \rightarrow 10$

$l=-18 \rightarrow 18$
Table 1

Selected geometric parameters $\left(\AA{ }^{\circ}\right)$.

\begin{tabular}{llll}
\hline $\mathrm{C} 1-\mathrm{C} 2$ & $1.397(2)$ & $\mathrm{C} 13-\mathrm{C} 14$ & $1.343(4)$ \\
$\mathrm{C} 1-\mathrm{C} 6$ & $1.390(2)$ & $\mathrm{C} 14-\mathrm{C} 15$ & $1.328(4)$ \\
$\mathrm{C} 1-\mathrm{C} 7$ & $1.477(2)$ & $\mathrm{C} 15-\mathrm{C} 16$ & $1.379(3)$ \\
$\mathrm{C} 2-\mathrm{O} 10$ & $1.371(2)$ & $\mathrm{C} 3-\mathrm{C} 4$ & $1.367(3)$ \\
$\mathrm{C} 2-\mathrm{C} 3$ & $1.389(3)$ & $\mathrm{C} 4-\mathrm{C} 5$ & $1.376(3)$ \\
$\mathrm{O} 10-\mathrm{C} 11$ & $1.387(2)$ & $\mathrm{C} 5-\mathrm{C} 6$ & $1.378(3)$ \\
$\mathrm{C} 11-\mathrm{C} 12$ & $1.346(3)$ & $\mathrm{C} 7-\mathrm{O} 9$ & $1.223(2)$ \\
$\mathrm{C} 11-\mathrm{C} 16$ & $1.372(4)$ & $\mathrm{C} 7-\mathrm{O} 8$ & $1.3015(18)$ \\
$\mathrm{C} 12-\mathrm{C} 13$ & $1.373(3)$ & & \\
$\mathrm{C} 2-\mathrm{C} 1-\mathrm{C} 6$ & $117.52(16)$ & $\mathrm{C} 12-\mathrm{C} 13-\mathrm{C} 14$ & $121.1(2)$ \\
$\mathrm{C} 2-\mathrm{C} 1-\mathrm{C} 7$ & $122.57(15)$ & $\mathrm{C} 13-\mathrm{C} 14-\mathrm{C} 15$ & $119.9(2)$ \\
$\mathrm{C} 6-\mathrm{C} 1-\mathrm{C} 7$ & $119.91(14)$ & $\mathrm{C} 14-\mathrm{C} 15-\mathrm{C} 16$ & $120.9(3)$ \\
$\mathrm{C} 1-\mathrm{C} 2-\mathrm{O} 10$ & $117.58(15)$ & $\mathrm{C} 15-\mathrm{C} 16-\mathrm{C} 11$ & $118.8(2)$ \\
$\mathrm{C} 1-\mathrm{C} 2-\mathrm{C} 3$ & $120.32(16)$ & $\mathrm{C} 2-\mathrm{C} 3-\mathrm{C} 4$ & $120.45(17)$ \\
$\mathrm{O} 10-\mathrm{C} 2-\mathrm{C} 3$ & $122.08(15)$ & $\mathrm{C} 3-\mathrm{C} 4-\mathrm{C} 5$ & $120.44(19)$ \\
$\mathrm{C} 2-\mathrm{O} 10-\mathrm{C} 11$ & $119.76(14)$ & $\mathrm{C} 4-\mathrm{C} 5-\mathrm{C} 6$ & $119.18(18)$ \\
$\mathrm{O} 10-\mathrm{C} 11-\mathrm{C} 12$ & $119.5(2)$ & $\mathrm{C} 1-\mathrm{C} 6-\mathrm{C} 5$ & $122.08(17)$ \\
$\mathrm{O} 10-\mathrm{C} 11-\mathrm{C} 16$ & $120.0(2)$ & $\mathrm{C} 1-\mathrm{C} 7-\mathrm{O} 9$ & $124.03(14)$ \\
$\mathrm{C} 12-\mathrm{C} 11-\mathrm{C} 16$ & $120.14(19)$ & $\mathrm{C} 1-\mathrm{C} 7-\mathrm{O} 8$ & $114.22(15)$ \\
$\mathrm{C} 11-\mathrm{C} 12-\mathrm{C} 13$ & $119.2(2)$ & $\mathrm{O} 9-\mathrm{C} 7-\mathrm{O} 8$ & $121.75(16)$ \\
\hline
\end{tabular}

Table 2

Hydrogen-bond geometry $\left(\AA,{ }^{\circ}\right)$.

\begin{tabular}{lcclc}
\hline$D-\mathrm{H} \cdots A$ & $D-\mathrm{H}$ & $\mathrm{H} \cdots A$ & $D \cdots A$ & $D-\mathrm{H} \cdots A$ \\
\hline O8-H1$\cdots \mathrm{O9}^{\mathrm{i}}$ & $0.90(2)$ & $1.75(2)$ & $2.651(2)$ & $175(2)$ \\
\hline \multicolumn{2}{l}{ Symmetry code: (i) $3-x, 1-y,-z}$.
\end{tabular}

$\mathrm{H}$ atoms were positioned geometrically and refined as riding groups, with $\mathrm{C}-\mathrm{H}=1.0 \AA$ and $U_{\text {iso }}(\mathrm{H})=1.2 U_{\text {eq }}(\mathrm{C})$, except for atom $\mathrm{H} 1$, which was located in a Fourier difference map and refined with an $\mathrm{O}-\mathrm{H}$ distance restraint of $0.90(5) \AA$ and a fixed $U_{\text {iso }}(\mathrm{H})=0.05 \AA^{2}$.

Data collection: $A P E X 2$ (Bruker, 2005); cell refinement: $A P E X 2$; data reduction: $A P E X 2$; $\operatorname{program}(\mathrm{s})$ used to solve structure: $S I R 92$ (Altomare et al., 1994); program(s) used to refine structure: CRYSTALS (Betteridge et al., 2003); molecular graphics: ORTEP3 for Windows (Farrugia, 1997) and MERCURY (Bruno et al., 2002); software used to prepare material for publication: CRYSTALS.

This paper is the result of an optional undergraduate class project entitled 'Frontiers of Crystallography', designed to show some of the sort of research that can be undertaken in crystallography. The data collection, structure solution, refinement and post-refinement analysis of the unknown title structure were all undertaken in parallel by the undergraduate students, who are all co-authors, and the collated information has resulted in this paper.

\section{References}

Allen, F. H. (2002). Acta Cryst. B58, 380-388.

Altomare, A., Cascarano, G., Giacovazzo, C., Guagliardi, A., Burla, M. C., Polidori, G. \& Camalli, M. (1994). J. Appl. Cryst. 27, 435.

Betteridge, P. W., Carruthers, J. R., Cooper, R. I., Prout, C. K. \& Watkin, D. J. (2003). J. Appl. Cryst. 36, 1487.

Bruker (2005). APEX2. Bruker AXS Inc., Madison, Wisconsin, USA.

Bruno, I. J., Cole, J. C., Edgington, P. R., Kessler, M., Macrae, C. F., McCabe, P., Pearson, J. \& Taylor, R. (2002). Acta Cryst. B58, 389-397.

Farrugia, L. J. (1997). J. Appl. Cryst. 30, 565.

Farrugia, L. J. (1999). J. Appl. Cryst. 32, 837-838.

Field, J. E. \& Venkataraman, D. (2002). Chem. Commun. pp. 306-307.

Sheldrick, G. M. (1996). SADABS. University of Göttingen, Germany. 


\section{supporting information}

Acta Cryst. (2005). E61, o2280-o2282［https://doi.org/10.1107/S1600536805019495]

\section{2-Phenoxybenzoic acid at room temperature}

Andrew Parkin, Suzanne M. Harte, Donnie Carmichael, Scott Currie, Lorna Drummond, Adam Haahr, Kevin Haggarty, Tracey Hunter, André Lamarque, Loretta Lawton, Craig Martin, Jennifer E. Mathieson, Jennifer S. Mathieson, Thomas McGlone, Julie McGregor, Liam McMillan, Louise Robertson, Robert Thatcher, Steven Vance and Chick C. Wilson

2-Phenoxybenzoic acid

Crystal data

$\mathrm{C}_{13} \mathrm{H}_{10} \mathrm{O}_{3}$

$M_{r}=214.22$

Triclinic, $P \overline{1}$

Hall symbol: -P 1

$a=5.2736(5) \AA$

$b=7.7366(6) \AA$

$c=13.6863(10) \AA$

$\alpha=89.184(6)^{\circ}$

$\beta=83.433(6)^{\circ}$

$\gamma=74.640(6)^{\circ}$

$V=534.84(8) \AA^{3}$

\section{Data collection}

Bruker APEX2

diffractometer

Graphite monochromator

$\varphi$ and $\omega$ scans

Absorption correction: multi-scan

(SADABS; Sheldrick, 1996)

$T_{\min }=0.98, T_{\max }=0.99$

8180 measured reflections

\section{Refinement}

Refinement on $F^{2}$

Least-squares matrix: full

$R\left[F^{2}>2 \sigma\left(F^{2}\right)\right]=0.054$

$w R\left(F^{2}\right)=0.164$

$S=0.93$

2565 reflections

148 parameters

1 restraint

Primary atom site location: structure-invariant direct methods
$Z=2$

$F(000)=224$

$D_{\mathrm{x}}=1.330 \mathrm{Mg} \mathrm{m}^{-3}$

Mo $K \alpha$ radiation, $\lambda=0.71073 \AA$

Cell parameters from 8180 reflections

$\theta=2-28^{\circ}$

$\mu=0.10 \mathrm{~mm}^{-1}$

$T=293 \mathrm{~K}$

Block, colourless

$0.30 \times 0.15 \times 0.10 \mathrm{~mm}$

2565 independent reflections

1516 reflections with $I>2 \sigma(I)$

$R_{\text {int }}=0.028$

$\theta_{\max }=28.5^{\circ}, \theta_{\min }=1.5^{\circ}$

$h=-6 \rightarrow 7$

$k=-10 \rightarrow 10$

$l=-18 \rightarrow 18$

Hydrogen site location: inferred from neighbouring sites

$\mathrm{H}$ atoms treated by a mixture of independent and constrained refinement

$w=1 /\left[\sigma^{2}\left(F^{2}\right)+0.08+0.08 P\right]$, where $P=\left[\max \left(F_{\mathrm{o}}^{2}, 0\right)+2 F_{\mathrm{c}}{ }^{2}\right] / 3$

$(\Delta / \sigma)_{\max }=0.000159$

$\Delta \rho_{\max }=0.38 \mathrm{e} \AA^{-3}$

$\Delta \rho_{\min }=-0.29$ e $\AA^{-3}$ 
Fractional atomic coordinates and isotropic or equivalent isotropic displacement parameters $\left(\AA^{2}\right)$

\begin{tabular}{|c|c|c|c|c|}
\hline & $x$ & $y$ & $z$ & $U_{\text {iso }} * / U_{\text {eq }}$ \\
\hline $\mathrm{C} 1$ & $1.0774(3)$ & $0.2871(2)$ & $0.12595(11)$ & 0.0538 \\
\hline $\mathrm{C} 2$ & $0.8810(4)$ & $0.3698(2)$ & $0.20060(13)$ & 0.0663 \\
\hline $\mathrm{O} 10$ & $0.8556(4)$ & 0.54655 (19) & $0.22196(13)$ & 0.1192 \\
\hline $\mathrm{C} 11$ & $0.6892(5)$ & $0.6279(2)$ & $0.30383(16)$ & 0.0787 \\
\hline $\mathrm{C} 12$ & $0.4462(5)$ & $0.7320(3)$ & $0.29248(17)$ & 0.0909 \\
\hline $\mathrm{C} 13$ & $0.2911(5)$ & $0.8241(4)$ & $0.3725(2)$ & 0.0970 \\
\hline $\mathrm{C} 14$ & $0.3793(5)$ & 0.8138 & 0.46118 (19) & 0.0911 \\
\hline $\mathrm{C} 15$ & $0.6204(6)$ & $0.7129(4)$ & 0.47235 (19) & 0.1149 \\
\hline $\mathrm{C} 16$ & $0.7826(5)$ & $0.6184(4)$ & $0.3938(2)$ & 0.1112 \\
\hline $\mathrm{C} 3$ & $0.7150(4)$ & 0.2753 & $0.24872(15)$ & 0.0760 \\
\hline $\mathrm{C} 4$ & $0.7430(4)$ & $0.1000(3)$ & $0.22415(16)$ & 0.0772 \\
\hline $\mathrm{C} 5$ & $0.9367(4)$ & $0.0147(3)$ & $0.15164(16)$ & 0.0781 \\
\hline C6 & 1.1009 (4) & $0.1085(2)$ & $0.10356(14)$ & 0.0673 \\
\hline $\mathrm{C} 7$ & $1.2568(3)$ & $0.3820(2)$ & $0.07145(11)$ & 0.0558 \\
\hline O9 & $1.2443(3)$ & $0.54022(17)$ & $0.08452(10)$ & 0.0788 \\
\hline $\mathrm{O} 8$ & $1.4326(3)$ & 0.28285 (19) & $0.00646(10)$ & 0.0819 \\
\hline H12 & 0.3789 & 0.7416 & 0.2267 & $0.1076^{*}$ \\
\hline H13 & 0.1095 & 0.9007 & 0.3647 & $0.1114^{*}$ \\
\hline H14 & 0.2637 & 0.8818 & 0.5187 & $0.1056^{*}$ \\
\hline $\mathrm{H} 15$ & 0.6847 & 0.7053 & 0.5386 & $0.1326^{*}$ \\
\hline H16 & 0.9651 & 0.5443 & 0.4023 & $0.1243^{*}$ \\
\hline $\mathrm{H} 3$ & 0.5743 & 0.3357 & 0.3018 & $0.0891^{*}$ \\
\hline $\mathrm{H} 4$ & 0.6217 & 0.0335 & 0.2590 & $0.0934^{*}$ \\
\hline $\mathrm{H} 5$ & 0.9583 & -0.1138 & 0.1341 & $0.0941 *$ \\
\hline H6 & 1.2412 & 0.0460 & 0.0508 & $0.0791 *$ \\
\hline H1 & $1.535(3)$ & $0.348(2)$ & $-0.0246(12)$ & $0.0500^{*}$ \\
\hline
\end{tabular}

Atomic displacement parameters $\left(\AA^{2}\right)$

\begin{tabular}{lllllll}
\hline & $U^{11}$ & $U^{22}$ & $U^{33}$ & $U^{12}$ & $U^{13}$ & $U^{23}$ \\
\hline C1 & $0.0574(9)$ & $0.0501(9)$ & $0.0474(8)$ & $-0.0055(7)$ & $0.0014(7)$ & $-0.0040(7)$ \\
C2 & $0.0805(12)$ & $0.0475(9)$ & $0.0618(10)$ & $-0.0113(8)$ & $0.0168(9)$ & $-0.0063(8)$ \\
O10 & $0.1579(16)$ & $0.0575(8)$ & $0.1224(13)$ & $-0.0398(9)$ & $0.0961(12)$ & $-0.0311(8)$ \\
C11 & $0.0982(15)$ & $0.0472(9)$ & $0.0814(14)$ & $-0.0263(10)$ & $0.0472(12)$ & $-0.0152(9)$ \\
C12 & $0.0975(17)$ & $0.0935(16)$ & $0.0768(14)$ & $-0.0243(14)$ & $0.0109(12)$ & $-0.0181(12)$ \\
C13 & $0.0789(15)$ & $0.0970(17)$ & $0.0991(18)$ & $-0.0059(12)$ & $0.0176(13)$ & $-0.0198(14)$ \\
C14 & $0.0976(17)$ & $0.0790(14)$ & $0.0869(16)$ & $-0.0231(12)$ & $0.0335(13)$ & $-0.0306(12)$ \\
C15 & $0.112(2)$ & $0.143(3)$ & $0.0750(16)$ & $-0.0165(19)$ & $0.0088(15)$ & $-0.0134(15)$ \\
C16 & $0.0877(17)$ & $0.113(2)$ & $0.108(2)$ & $0.0039(15)$ & $0.0198(15)$ & $0.0077(16)$ \\
C3 & $0.0873(14)$ & $0.0593(11)$ & $0.0732(12)$ & $-0.0190(10)$ & $0.0252(10)$ & $-0.0048(9)$ \\
C4 & $0.0891(14)$ & $0.0625(12)$ & $0.0803(13)$ & $-0.0275(10)$ & $0.0066(11)$ & $0.0009(10)$ \\
C5 & $0.0920(15)$ & $0.0551(11)$ & $0.0859(14)$ & $-0.0215(10)$ & $0.0018(11)$ & $-0.0131(10)$ \\
C6 & $0.0723(12)$ & $0.0571(10)$ & $0.0664(11)$ & $-0.0115(9)$ & $0.0051(9)$ & $-0.0151(8)$ \\
C7 & $0.0576(9)$ & $0.0532(9)$ & $0.0481(9)$ & $-0.0041(7)$ & $0.0046(7)$ & $-0.0082(7)$ \\
O9 & $0.0870(9)$ & $0.0578(8)$ & $0.0806(9)$ & $-0.0182(6)$ & $0.0350(7)$ & $-0.0159(6)$
\end{tabular}


$\begin{array}{lllllll}\text { O8 } & 0.0886(10) & 0.0656(8) & 0.0801(9) & -0.0197(7) & 0.0387(8) & -0.0198(7)\end{array}$

Geometric parameters $(\AA, \stackrel{o}{)}$

\begin{tabular}{|c|c|c|c|}
\hline $\mathrm{C} 1-\mathrm{C} 2$ & $1.397(2)$ & $\mathrm{C} 15-\mathrm{C} 16$ & $1.379(3)$ \\
\hline $\mathrm{C} 1-\mathrm{C} 6$ & $1.390(2)$ & $\mathrm{C} 15-\mathrm{H} 15$ & 1.000 \\
\hline $\mathrm{C} 1-\mathrm{C} 7$ & $1.477(2)$ & $\mathrm{C} 16-\mathrm{H} 16$ & 1.000 \\
\hline $\mathrm{C} 2-\mathrm{O} 10$ & $1.371(2)$ & $\mathrm{C} 3-\mathrm{C} 4$ & $1.367(3)$ \\
\hline $\mathrm{C} 2-\mathrm{C} 3$ & $1.389(3)$ & $\mathrm{C} 3-\mathrm{H} 3$ & 1.000 \\
\hline $\mathrm{O} 10-\mathrm{C} 11$ & $1.387(2)$ & $\mathrm{C} 4-\mathrm{C} 5$ & $1.376(3)$ \\
\hline $\mathrm{C} 11-\mathrm{C} 12$ & $1.346(3)$ & $\mathrm{C} 4-\mathrm{H} 4$ & 1.000 \\
\hline $\mathrm{C} 11-\mathrm{C} 16$ & $1.372(4)$ & $\mathrm{C} 5-\mathrm{C} 6$ & $1.378(3)$ \\
\hline $\mathrm{C} 12-\mathrm{C} 13$ & $1.373(3)$ & $\mathrm{C} 5-\mathrm{H} 5$ & 1.000 \\
\hline $\mathrm{C} 12-\mathrm{H} 12$ & 1.000 & C6- $\mathrm{H} 6$ & 1.000 \\
\hline $\mathrm{C} 13-\mathrm{C} 14$ & $1.343(4)$ & $\mathrm{C} 7-\mathrm{O} 9$ & $1.223(2)$ \\
\hline $\mathrm{C} 13-\mathrm{H} 13$ & 1.000 & $\mathrm{C} 7-\mathrm{O} 8$ & $1.3015(18)$ \\
\hline $\mathrm{C} 14-\mathrm{C} 15$ & $1.328(4)$ & $\mathrm{O} 8-\mathrm{H} 1$ & $0.901(17)$ \\
\hline $\mathrm{C} 14-\mathrm{H} 14$ & 1.000 & & \\
\hline $\mathrm{C} 2-\mathrm{C} 1-\mathrm{C} 6$ & $117.52(16)$ & $\mathrm{C} 16-\mathrm{C} 15-\mathrm{H} 15$ & 119.7 \\
\hline $\mathrm{C} 2-\mathrm{C} 1-\mathrm{C} 7$ & $122.57(15)$ & $\mathrm{C} 15-\mathrm{C} 16-\mathrm{C} 11$ & $118.8(2)$ \\
\hline $\mathrm{C} 6-\mathrm{C} 1-\mathrm{C} 7$ & $119.91(14)$ & $\mathrm{C} 15-\mathrm{C} 16-\mathrm{H} 16$ & 120.6 \\
\hline $\mathrm{C} 1-\mathrm{C} 2-\mathrm{O} 10$ & $117.58(15)$ & $\mathrm{C} 11-\mathrm{C} 16-\mathrm{H} 16$ & 120.6 \\
\hline $\mathrm{C} 1-\mathrm{C} 2-\mathrm{C} 3$ & $120.32(16)$ & $\mathrm{C} 2-\mathrm{C} 3-\mathrm{C} 4$ & $120.45(17)$ \\
\hline $\mathrm{O} 10-\mathrm{C} 2-\mathrm{C} 3$ & $122.08(15)$ & $\mathrm{C} 2-\mathrm{C} 3-\mathrm{H} 3$ & 119.8 \\
\hline $\mathrm{C} 2-\mathrm{O} 10-\mathrm{C} 11$ & $119.76(14)$ & $\mathrm{C} 4-\mathrm{C} 3-\mathrm{H} 3$ & 119.8 \\
\hline $\mathrm{O} 10-\mathrm{C} 11-\mathrm{C} 12$ & $119.5(2)$ & $\mathrm{C} 3-\mathrm{C} 4-\mathrm{C} 5$ & 120.44 (19) \\
\hline $\mathrm{O} 10-\mathrm{C} 11-\mathrm{C} 16$ & $120.0(2)$ & $\mathrm{C} 3-\mathrm{C} 4-\mathrm{H} 4$ & 119.8 \\
\hline $\mathrm{C} 12-\mathrm{C} 11-\mathrm{C} 16$ & $120.14(19)$ & $\mathrm{C} 5-\mathrm{C} 4-\mathrm{H} 4$ & 119.8 \\
\hline $\mathrm{C} 11-\mathrm{C} 12-\mathrm{C} 13$ & $119.2(2)$ & $\mathrm{C} 4-\mathrm{C} 5-\mathrm{C} 6$ & $119.18(18)$ \\
\hline $\mathrm{C} 11-\mathrm{C} 12-\mathrm{H} 12$ & 120.4 & $\mathrm{C} 4-\mathrm{C} 5-\mathrm{H} 5$ & 120.4 \\
\hline $\mathrm{C} 13-\mathrm{C} 12-\mathrm{H} 12$ & 120.4 & $\mathrm{C} 6-\mathrm{C} 5-\mathrm{H} 5$ & 120.4 \\
\hline $\mathrm{C} 12-\mathrm{C} 13-\mathrm{C} 14$ & $121.1(2)$ & $\mathrm{C} 1-\mathrm{C} 6-\mathrm{C} 5$ & $122.08(17)$ \\
\hline $\mathrm{C} 12-\mathrm{C} 13-\mathrm{H} 13$ & 119.6 & $\mathrm{C} 1-\mathrm{C} 6-\mathrm{H} 6$ & 119.0 \\
\hline $\mathrm{C} 14-\mathrm{C} 13-\mathrm{H} 13$ & 119.3 & $\mathrm{C} 5-\mathrm{C} 6-\mathrm{H} 6$ & 118.9 \\
\hline $\mathrm{C} 13-\mathrm{C} 14-\mathrm{C} 15$ & $119.9(2)$ & $\mathrm{C} 1-\mathrm{C} 7-\mathrm{O} 9$ & $124.03(14)$ \\
\hline $\mathrm{C} 13-\mathrm{C} 14-\mathrm{H} 14$ & 120.0 & $\mathrm{C} 1-\mathrm{C} 7-\mathrm{O} 8$ & $114.22(15)$ \\
\hline $\mathrm{C} 15-\mathrm{C} 14-\mathrm{H} 14$ & 120.1 & $\mathrm{O} 9-\mathrm{C} 7-\mathrm{O} 8$ & $121.75(16)$ \\
\hline $\mathrm{C} 14-\mathrm{C} 15-\mathrm{C} 16$ & $120.9(3)$ & $\mathrm{C} 7-\mathrm{O} 8-\mathrm{H} 1$ & $110.1(10)$ \\
\hline $\mathrm{C} 14-\mathrm{C} 15-\mathrm{H} 15$ & 119.5 & & \\
\hline
\end{tabular}

Hydrogen-bond geometry $(\AA, \stackrel{o}{)}$

\begin{tabular}{lllll}
\hline$D-\mathrm{H} \cdots A$ & $D-\mathrm{H}$ & $\mathrm{H} \cdots A$ & $D \cdots A$ & $D-\mathrm{H} \cdots A$ \\
\hline $\mathrm{O} 8-\mathrm{H} 1 \cdots \mathrm{O} 9 \mathrm{i}$ & $0.90(2)$ & $1.75(2)$ & $2.651(2)$ & $175(2)$ \\
\hline
\end{tabular}

Symmetry code: (i) $-x+3,-y+1,-z$. 